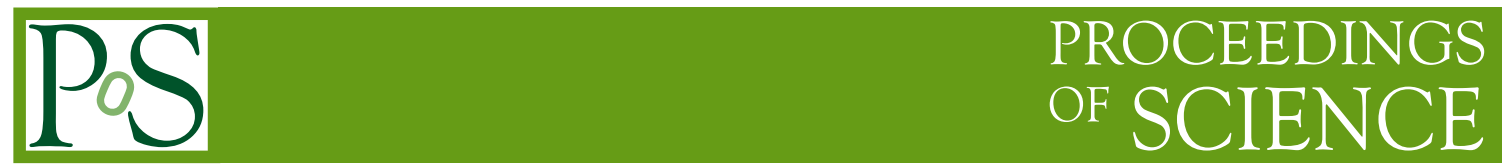

\title{
All-plus helicity off-shell gauge invariant multigluon amplitudes at one loop
}

\author{
Etienne Blanco, ${ }^{a, *}$ Andreas van Hameren, ${ }^{a}$ Piotr Kotko ${ }^{b}$ and Krzysztof Kutak ${ }^{a}$ \\ ${ }^{a}$ Institute of Nuclear Physics, Polish Academy of Sciences, \\ Radzikowskiego 152, 31-342 Krakow, Poland \\ ${ }^{b}$ AGH University Of Science and Technology, Physics Faculty, \\ Mickiewicza 30, 30-059 Krakow, Poland \\ E-mail: etienne.blanco@ifj.edu.pl
}

We calculate one loop scattering amplitudes for arbitrary number of positive helicity on-shell gluons and one off-shell gluon treated within the quasi-multi Regge kinematics. The result is fully gauge invariant and possesses the correct on-shell limit. Our method is based on embedding the off-shell process, together with contributions needed to retain gauge invariance, in a bigger fully on-shell process with auxiliary quark or gluon line.

*** The European Physical Society Conference on High Energy Physics (EPS-HEP2021), *** *** 26-30 July $2021 * * *$

*** Online conference, jointly organized by Universität Hamburg and the research center DESY ***

${ }^{*}$ Speaker 


\section{Introduction}

One of the key results in the high energy limit of Quantum Chromodynamics (QCD), also called the small-x regime, is the so-called $k_{T}$-factorization (or high energy factorization). In this approach the cross section is expressed as:

$$
\begin{array}{r}
d \sigma(a b \rightarrow \mathcal{X})=\sum_{i, j} \int \frac{d^{2} \mathbf{k}_{T, i}}{\pi} \frac{d^{2} \mathbf{k}_{T, j}}{\pi} d x_{i} d x_{j} f_{i, a}\left(x_{i}, \mathbf{k}_{T, i}, \mu\right) f_{j, b}\left(x_{j}, \mathbf{k}_{T, j}, \mu\right) \times \\
\times d \hat{\sigma}\left(i^{*} j^{*} \rightarrow \mathcal{X}\right)\left(x_{i}, \mathbf{k}_{T, i}, x_{j}, \mathbf{k}_{T, j}, \mu\right)
\end{array}
$$

Unlike collinear factorization, both the hard part and the soft hadronic part depends on the parton transverse momenta. The Transverse Momentum Dependant parton distribution functions (TMDs) follow Balitsky-Fadin-Kuraev-Lipatov (BFKL) evolution equation and the hard matrix elements are defined with momenta of the partons that in general are off-shell.

Our focus is the calculation of scattering amplitudes, where only one gluon is off-shell. Such amplitudes are used to describe forward particle production. In high energy kinematics, the momentum of the off-shell gluon has the form :

$$
k^{\mu}=x p^{\mu}+k_{T}^{\mu},
$$

where $p^{\mu}$ is the light-like momentum typically associated with the colliding hadron, $x$ is the fraction of this momentum carried by the scattering parton, and $k_{T}^{\mu}$ is the transverse component satisfying $k_{T} \cdot p=0$. The off-shell gluon couples eikonally, i.e. its vector index is projected onto $p^{\mu}$ (the propagator is included in the amplitude), see Fig. 1. However, the standard diagrams contributing to such amplitudes are not gauge invariant. A proper definition of these off-shell amplitudes can be obtained within the Lipatov's high energy effective action $[1,2]$ or by construction, calculating explicitly terms required to recover gauge invariance (and also proper soft and collinear behavior). The latter method can be approached in several ways: using the Ward identities [3], using matrix of straight infinite Wilson lines [4] or embedding the off-shell process into a bigger on-shell one [5]. We will use the embedding method and review it in Section 2. This method has the advantage to be based on on-shell results (which calculations are automatized at one-loop) and has already proved to be effective at tree level. Also, it is implemented in the Monte Carlo generator KaTie [6]. A proof of concept of its generalization at one-loop level has already been given in [7]. Then, in this work, we aim at validating this concept by applying the embedding method at one-loop and verifying the correct behaviour of the obtained amplitudes. As a first step, we have chosen to study off-shell amplitude with one off-shell gluon and any number $n$ of on-shell gluons of the same helicity, say 'plus' (considering outgoing momenta). We present our most general result, i.e. the all-plus helicity gluons and one off-shell gluon in Section 3 and the verification of its on-shell behaviour in Section 4.

\section{The method}

First, we present the method used to calculate amplitudes with one off-shell leg (presented first in [5]). As mentioned in the introduction, the main idea to guarantee gauge invariance and the 


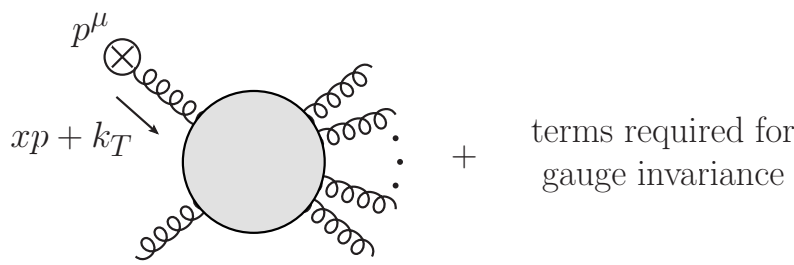

Figure 1: In high energy factorization for forward jets the multigluon amplitude has one incoming momentum off mass shell, with the off-shell propagator projected onto light-like momentum $p^{\mu}$ (typically the momentum of the hadron to which the gluon couples). The momentum of the off-shell leg has only one longitudinal component in the high energy kinematics. Such amplitude is in general not gauge invariant and additional terms are required to define it properly.

proper soft and collinear behaviour of the off-shell amplitude is to embed it into a larger on-shell one. In this method, we consider the amplitude with an auxiliary quark-antiquark pair as our on-shell bigger process. Schematically, the method can be summarized as (see also Fig. 2) :

$$
\lim _{\Lambda \rightarrow \infty}\left(\frac{x\left|k_{T}\right|}{g_{s} \Lambda} \mathcal{A}\left(\bar{q}\left(k_{1}\right) q\left(k_{2}\right) \mathcal{X}\right)\right)=\mathcal{A}^{*}\left(g^{*}(k) \mathcal{X}\right),
$$

where $\mathcal{X}$ stands for other on-shell particles involved in the hard scattering process and $\Lambda$ is a real parameter parametrizing momenta of the auxiliary quark pair (see below). The gauge invariant off-shell amplitude is denoted $\mathcal{A}^{*}$. The momenta of the auxiliary quarks are taken to be the following :

$$
\begin{aligned}
& k_{1}^{\mu}=\Lambda p^{\mu}+\alpha q^{\mu}+\beta k_{T}^{\mu}, \\
& k_{2}^{\mu}=k^{\mu}-k_{1}^{\mu},
\end{aligned}
$$

where

$$
\alpha=\frac{-\beta^{2} k_{T}^{2}}{2 \Lambda p \cdot q} \quad, \quad \beta=\frac{1}{1+\sqrt{1-x / \Lambda}}
$$

and $q^{\mu}$ is an arbitrary light-like momentum such that $q \cdot k_{T}=0, q \cdot p>0$. Note, that $k_{1}^{\mu}$ and $k_{2}^{\mu}$ are light-like and they satisfy $k_{1}^{\mu}+k_{2}^{\mu}=k^{\mu}$, where the latter is the momentum of the off-shell gluon as defined in Eq. (2). In the limit $\Lambda \rightarrow \infty$ the coupling of gluons to the quark line becomes eikonal, consistent with the high energy limit. The factor $1 / g_{s}$ in Eq. (3) is to correct the power of the coupling, and the factor $x\left|k_{T}\right|$ is for the correct matching to $k_{T}$-dependent PDFs in a cross section. In particular, the factor $\left|k_{T}\right|$ makes sure the amplitude is finite for $\left|k_{T}\right| \rightarrow 0$.

In what follows, we shall call the above kinematics (together with taking the limit $\Lambda \rightarrow \infty$ ) the " $\Lambda$ prescription". Applying it to an amplitude with auxiliary partons gives the gauge invariant off-shell amplitude.

Equivalently, the "embedding" method described above can be used with auxiliary gluon pair, instead of the quark-antiquark pair. Indeed, the color decomposition for $(n-2)$-gluon amplitude with a quark-antiquark pair can be projected onto $(n-1)$-gluon amplitude by a contraction with $\left(t^{a_{*}}\right)_{j i}$, where $a_{*}$ represents the color index of the off-shell gluon. Then, this is a matter of picking the right color terms (see [7] for details). In [7] it has been shown that at tree level, the partial amplitudes obtained using different pairs of auxiliary partons are identical. 


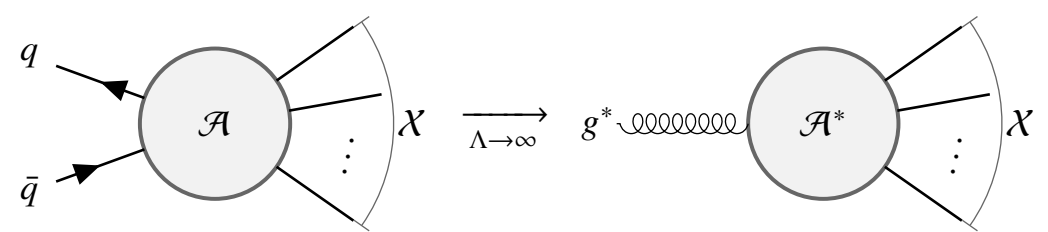

Figure 2: Gauge invariant off-shell amplitudes can be obtained by considering a process with an auxiliary quark-antiquark pair, with momenta parametrized in terms of a parameter $\Lambda$ in such a way, that upon taking the limit $\Lambda \rightarrow \infty$ the coupling to the quark line becomes eikonal and the momentum of the off-shell gluon has the high energy form (see Eq. (2)).

\section{All-plus off-shell gauge invariant amplitudes at NLO}

In the following section we shall derive the expression for one-loop amplitude for one off-shell gluon and $n-1$ on-shell gluons with all helicities positive. To this end, we need the one loop amplitude for a quark-antiquark pair and $n-1$ positive helicity gluons. A suitable expression has been derived in [8]. It reads

$$
\begin{aligned}
\mathcal{A}_{n+1}^{(1)}\left(1_{\bar{q}}^{-}, 2_{q}^{+}, 3^{+}, \cdots,(n+1)^{+}\right)= & \frac{i g_{s}^{n+1}}{32 \pi^{2}}\left(1+\frac{1}{N_{c}^{2}}\right) \frac{\sum_{l=3}^{n}\left\langle 1\left|K_{2 \ldots l} l K_{l}\right| 1\right\rangle}{\langle 23\rangle \cdots\langle(n+1) 1\rangle} \\
& +\frac{i g_{s}^{n+1}}{48 \pi^{2}}\left(1+\frac{n_{s}-n_{f}}{N_{c}}\right) \frac{S_{1}+S_{2}}{\langle 12\rangle\langle 23\rangle \cdots\langle(n+1) 1\rangle},
\end{aligned}
$$

with

$$
\begin{aligned}
S_{1}=\sum_{j=3}^{n} \frac{\langle 2 j\rangle\langle 1(j+1)\rangle\left\langle 1\left|\mathbb{K}_{j, j+1} \mathbb{K}_{(j+1) \cdots(n+1)}\right| 1\right\rangle}{\langle j(j+1)\rangle}, \\
S_{2}=\sum_{j=3}^{n-1} \sum_{l=j+1}^{n} \frac{\left\langle 1\left|\mathbb{K}_{j \cdots l} \mathbb{K}_{(l+1) \cdots(n+1)}\right| 1\right\rangle^{2}\left\langle 2\left|\mathbb{K}_{j \cdots l} \mathbb{K}_{(l+1) \cdots(n+1)}\right| 1\right\rangle}{\left\langle 1\left|\mathbb{K}_{(l+1) \cdots(n+1)} \mathbb{K}_{j \cdots l}\right|(j-1)\right\rangle\left\langle 1\left|\mathbb{K}_{(l+1) \cdots(n+1)} \mathbb{K}_{j \cdots l}\right| j\right\rangle} \\
\quad \times \frac{\langle(j-1) j\rangle\langle l(l+1)\rangle\left\langle 1\left|\mathbb{K}_{2 \cdots(j-1)}[\mathcal{F}(j, l)]^{2} \mathbb{K}_{(l+1) \cdots(n+1)}\right| 1\right\rangle}{\left\langle 1\left|\mathbb{K}_{2 \cdots(j-1)} \mathbb{K}_{j \cdots l}\right| l\right\rangle\left\langle 1\left|\mathbb{K}_{2 \cdots(j-1)} \mathbb{K}_{j \cdots l}\right|(l+1)\right\rangle s_{j \cdots l}},
\end{aligned}
$$

where

$$
\mathcal{F}(j, l)=\sum_{i=j}^{l-1} \sum_{m=i+1}^{l} k_{i} k_{m} .
$$

After applying the $\Lambda$ prescription we find that the term with the factor $\left(1+\frac{1}{N_{c}^{2}}\right)$ is of the order $\Lambda^{-1}$, whereas the other term is of order 1 and is the one contributing to the off-shell amplitude. Eventually, we obtain the following expression for the off-shell amplitude:

$$
\mathcal{A}_{n}^{*(1)}\left(g^{*}, 3^{+}, \cdots,(n+1)^{+}\right)=\frac{i g_{s}^{n} x\left|k_{T}\right|}{48 \pi^{2}}\left(1+\frac{n_{s}-n_{f}}{N_{c}}\right) \frac{U_{1}^{*}+U_{2}^{*}+U_{3}^{*}}{\kappa^{*}\langle p 3\rangle\langle 34\rangle \cdots\langle n p\rangle},
$$


with

$$
\begin{aligned}
U_{1}^{*}=\sum_{j=3}^{n} \frac{\langle p j\rangle\langle p(j+1)\rangle\left\langle p\left|\mathbb{K}_{j, j+1} \mathbb{K}_{(j+1) \cdots(n+1)}\right| p\right\rangle}{\langle j(j+1)\rangle}, \\
U_{2}^{*}=\sum_{j=4}^{n-1} \sum_{l=j+1}^{n} \frac{\left\langle p\left|\mathbb{K}_{j \cdots l} \mathbb{K}_{(l+1) \cdots(n+1)}\right| p\right\rangle^{3}}{\left\langle p\left|\mathbb{K}_{(l+1) \cdots(n+1)} \mathbb{K}_{j \cdots l}\right|(j-1)\right\rangle\left\langle p\left|\mathbb{K}_{(l+1) \cdots(n+1)} \mathbb{K}_{j \cdots l}\right| j\right\rangle} \\
\quad \times \frac{\langle(j-1) j\rangle\langle l(l+1)\rangle\left\langle p\left|\mathbb{K}_{3 \cdots(j-1)}^{\prime}[\mathcal{F}(j, l)]^{2} \mathbb{K}_{(l+1) \cdots(n+1)}\right| p\right\rangle}{\left\langle p\left|\mathbb{K}_{3 \cdots(j-1)} \mathbb{K}_{j \cdots l}\right| l\right\rangle\left\langle p\left|\mathbb{K}_{3 \cdots(j-1)} \mathbb{K}_{j \cdots l}\right|(l+1)\right\rangle s_{j \cdots l}}, \\
U_{3}^{*}=\sum_{l=4}^{n} \frac{\left\langle p\left|\mathbb{K}_{3 \cdots l} \mathbb{K}_{(l+1) \cdots(n+1)}\right| p\right\rangle^{3}}{\left\langle p\left|\mathbb{K}_{(l+1) \cdots(n+1)} \mathbb{K}_{3 \cdots l}\right| p\right\rangle\left\langle p\left|\mathbb{K}_{(l+1) \cdots(n+1)} \mathbb{K}_{3 \cdots l}\right| 3\right\rangle} \\
\quad \times \frac{\langle p 3\rangle\langle l(l+1)\rangle\left[p\left|[\mathcal{F}(3, l)]^{2} \mathbb{K}_{(l+1) \cdots(n+1)}\right| p\right\rangle}{\kappa^{*}\left[p\left|\mathbb{K}_{3 \cdots l}\right| l\right\rangle\left[p\left|\mathbb{K}_{3 \cdots l}\right|(l+1)\right\rangle s_{3 \cdots l}} .
\end{aligned}
$$

\section{Validating the result}

Now that we have our off-shell amplitude, some properties have to be checked. First, we want to verify that applying the $\Lambda$ prescription with either an auxiliary quark line or an auxiliary gluon line leads to the same result. The on-shell $n$-point gluonic amplitude is taken from [8] and applying the $\Lambda$-prescription to it leads to the same result as in Eq. Eq. (9) (details are given in [9].

Next, we need to verify the on-shell limit of the obtained amplitude, i.e. the limit $\left|k_{T}\right| \rightarrow 0$. In this limit, we expect our off-shell amplitude to express as a sum of two on-shell amplitude where the off-shell gluon tends to an on-shell one with either positive or negative helicity (see justification in $[9,10])$. More explicitly :

$$
\lim _{\left|k_{T}\right| \rightarrow 0} \mathcal{A}_{n}^{*(0)}\left(g^{*} \mathcal{X}\right)=\frac{\left|k_{T}\right|}{\kappa^{*}} \mathcal{A}_{n}^{(0)}\left(g^{-} \mathcal{X}\right)+\frac{\left|k_{T}\right|}{\kappa} \mathcal{A}_{n}^{(0)}\left(g^{+} \mathcal{X}\right),
$$

We now verify that the same limit appears for the one-loop $n$-point all-plus amplitudes we obtained in Section 3. One can notice that $U_{1}^{*} \underset{\left|k_{T}\right| \rightarrow 0}{\longrightarrow} T^{1}$ and $U_{2}^{*} \underset{\left|k_{T}\right| \rightarrow 0}{\longrightarrow} T^{2}$, which implies

$$
\begin{aligned}
\lim _{\left|k_{T}\right| \rightarrow 0} \mathcal{A}_{n}^{*(1)}\left(g^{*}, 3^{+}, \cdots,(n+1)^{+}\right)= & \frac{\left|k_{T}\right|}{\kappa^{*}} \mathcal{A}_{n}^{(1)}\left(x p^{-}, 3^{+}, \cdots,(n+1)^{+}\right) \\
& +\frac{i g_{s}^{n} x}{48 \pi^{2}} \frac{\lim _{k_{T} \rightarrow 0}\left(U_{3}^{*}\left|k_{T}\right| / \kappa^{*}\right)}{\langle p 3\rangle\langle 34\rangle \cdots\langle(n+1) p\rangle} .
\end{aligned}
$$

So we already have the contribution from the amplitude with negative helicity gluon (the first term in the expression above). It can be demonstrated that the second term actually correspond to the amplitude with positive helicity gluon (but this is much less trivial, see the demonstration in [9]) .

For $n=3$, we also verified that our result was in agreement with Lipatov's high energy effective action calculations (comparing it with results presented in [11]). 


\section{Conclusion}

We presented a method to calculate scattering amplitudes with one off-shell gluon. This method was already proved to be effective at tree level and in the present contribution we show that it works at one loop level for all-plus helicity gluon off-shell amplitudes. The obtained amplitude has the correct on-shell limit. Also, we verified that the embedding method works both with auxiliary quark and gluon line, giving the same result for the present amplitude (similarily to the tree-level case).

The generalization to one loop amplitudes with other helicity configurations (that are non-finite) is in progress. We have observed that applying the $\Lambda$ prescription and integrating loop momenta within dimensional regularization do not commute, which is the main obstacle towards a complete generalization of our method at one-loop level. Also the equivalence between results obtained with auxiliary quarks and auxiliary gluons does not hold but the difference between both approaches seems to be universal.

\section{References}

[1] L. N. Lipatov, Gauge invariant effective action for high-energy processes in $Q C D, N u c l$. Phys. B452 (1995) 369-400, [hep-ph/9502308].

[2] E. N. Antonov, L. N. Lipatov, E. A. Kuraev and I. O. Cherednikov, Feynman rules for effective Regge action, Nucl. Phys. B721 (2005) 111-135, [hep-ph/0411185].

[3] A. van Hameren, P. Kotko and K. Kutak, Multi-gluon helicity amplitudes with one off-shell leg within high energy factorization, JHEP 12 (2012) 029, [1207 . 3332].

[4] P. Kotko, Wilson lines and gauge invariant off-shell amplitudes, JHEP 07 (2014) 128, [1403.4824].

[5] A. van Hameren, P. Kotko and K. Kutak, Helicity amplitudes for high-energy scattering, JHEP 01 (2013) 078, [1211.0961].

[6] A. van Hameren, KaTie : For parton-level event generation with $k_{T}$-dependent initial states, Comput. Phys. Commun. 224 (2018) 371-380, [1611.00680].

[7] A. van Hameren, Calculating off-shell one-loop amplitudes for $k_{T}$-dependent factorization: a proof of concept, 1710.07609 .

[8] Z. Bern, L. J. Dixon and D. A. Kosower, Last of the finite loop amplitudes in qcd, Physical Review D 72 (2005) 125003.

[9] E. Blanco, A. van Hameren, P. Kotko and K. Kutak, All-plus helicity off-shell gauge invariant multigluon amplitudes at one loop, Journal of High Energy Physics 2020 (Dec, 2020) .

[10] A. van Hameren, BCFW recursion for off-shell gluons, JHEP 07 (2014) 138, [1404. 7818].

[11] M. Nefedov, One-loop corrections to multiscale effective vertices in the eft for multi-regge processes in qcd, 1905.01105. 